\title{
Development of Automatic Mold Shot Measurement and Management System for Smart Factory
}

\author{
Hyun-Jun Shin, Sung-Jin Kim, Min-Ho Jeon, Chang-Heon Oh \\ Department of Electrical, Electronics and Communication Engineering, Korea University of Technology and Education, \\ Chungcheongnam-do, Korea
}

\begin{tabular}{l}
\hline \hline Article Info \\
\hline Article history: \\
Received Aug 12, 2016 \\
Revised Oct 20, 2016 \\
Accepted Nov 8, 2016 \\
\hline
\end{tabular}

Keyword:

Automation system

Industrial 4.0

Injection mold management

Internet of things

Smart factory

\begin{abstract}
Many small- and medium-sized car-part manufacturers are either still managing their mold manually or rarely managing it, and therefore, experience significant manufacturing cost and loss in time. In such a situation, a module has been developed in the present work which can count the number of mold used. Such a module is extremely important for small and medium-sized enterprises (SMEs) applying which in the production line they will be able to manage the mold life cycle and improve product quality. This is expected to have both direct and indirect effects on their business activities. The developed system uses a photo sensor, distance measurement sensor, Atmega128 MCU, tablet pc and Bluetooth communication module. The actual module developed in this study was set up on a molding equipment for test and data were collected using an existing tablet PC. The test showed that the number of shots increased when the upper mold touched the lower mold. The maximum and minimum value between the upper and lower molds could be adjusted with the automatic mold shot measurement and management system. Therefore, any molding equipment with various upper-lower gaps will be able to apply the newly developed system.
\end{abstract}

Copyright $@ 2016$ Institute of Advanced Engineering and Science. All rights reserved.

\section{Corresponding Author:}

Chang-Heon Oh,

Department of Electrical, Electronics and Communication Engineering,

Korea University of Technology and Education ,

Gajeon-Ri-307, Byeongcheon-myeon, Dongnam-gu, Cheonan-si, Chungcheongnam-do, Korea.

Email: choh@koreatech.ac.kr

\section{INTRODUCTION}

The term "Industry 4.0" refers to a strategy of German manufacturing industries in which ICT is combined with out-out efforts in the face of social, technological, economic, ecological and political change. It is aimed to create a smart factory that will use ICT technologies actively, such as Internet of Things (IoT), corporate software, location information, security, cloud, big data and virtual reality. Smart manufacturing of the smart factory connects facilities and processing of an individual factory, and shares and uses all production information by combining ICT with traditional manufacturing, making it possible to achieve optimal production and operation. At the same time, it connects related factories to establish a production system which will allow continued collaboration through extension of this concept. Though the Republic of Korea is strong in manufacturing, it has an insignificant number of smart factories. It also needs innovation for competency in hardware and software technology, in particular, which is the base technology of a smart factory. Although Korea has excellent IT infrastructure, its companies have wide gaps between themselves. Additionally, the small and medium-sized enterprises have weak capacity and technological prowess [1-3].

Mold management system refers to the one that records information such as current location and condition of a forming mold when various parts are manufactured in the production. In this regard, car-part 
manufacturers are either still managing their mold manually or does not manage it at all which eventually leads to significant manufacturing cost and loss in time in supplying their goods [4-7].

In the current work, a module has been developed that can count the number of molds used in real time, help small- and medium-sized enterprises manage the mold life-cycle, and improve product quality through its application to the production line. This is expected to have both direct and indirect effects on their business.

\section{DEVELOPMENT OF AUTOMATIC MOLD SHOT MEASUREMENT AND MANAGEMENT SYSTEM}

Although a number of molding equipment are available, upper mold and lower mold contacts to produce injection molding are not common. Contact of the two molds is called shot and the mold is graded by the number of shots and the equipment should go through regular maintenance and repair when the shots are used up.

Table 1 shows regular checkup cycle of molds using mold rating. The life cycle of a mold declines with increasing number of shots. Therefore, regular maintenance is needed after a certain number of shots and repair should be done after the maximum shot. After maintenance or repair work, the mold is graded through rating. To note, recording the shots manually causes significant loss in time and money to supply parts. Therefore, automatic shot counting and management system is both useful and important.

Table 1. Regular Maintenance Cycle using Mold Rating

\begin{tabular}{ccccccc}
\hline $\begin{array}{c}\text { Guarantee } \\
\text { shot }\end{array}$ & Ratio $(\%)$ & $\begin{array}{c}\text { Minimum } \\
\text { shot }\end{array}$ & $\begin{array}{c}\text { Maximum } \\
\text { shot }\end{array}$ & Grade & Check period & $\begin{array}{c}\text { Check the number of } \\
\text { grades }\end{array}$ \\
\hline & 30 & - & 150,000 & A & 30,000 & 5 \\
500,000 & 50 & 150,000 & 270,000 & B & 25,000 & 5 \\
& 80 & 270,000 & 395,000 & C & 20,000 & 6 \\
& 80 excess & 395,000 & 500,000 & D & 15,000 & 7 over \\
\hline
\end{tabular}

As shown in Figure 1, a module uses photo sensor and distance measurement sensor to check contacts of the upper and lower molds and count the shots. The number of shots counted are then sent to a tablet pc via Bluetooth, and the tablet in turn sends the shots to the server, which helps managers monitor the mold usage efficiently. The photo sensor can detect objects in the front. It detects the existence of shaft put on the lower mold, and if the shaft is placed, the upper mold is ready to go down. Distance measurement sensor can measure distance of objects in the front. This sensor measures the distance between the upper mold and lower mold and indicates whether there has been a shot.

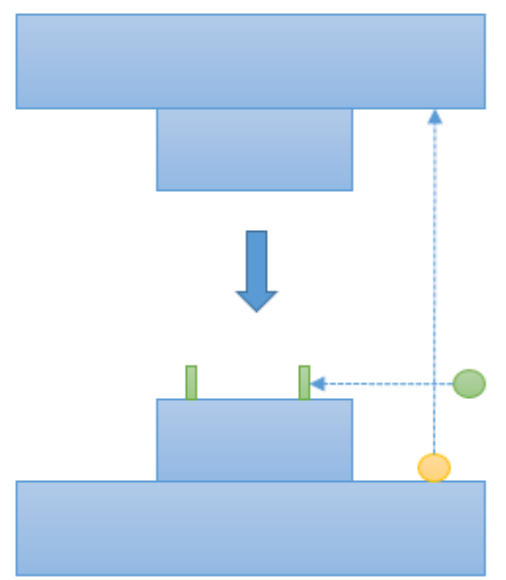

Distance measurement sensor

Photo sensor

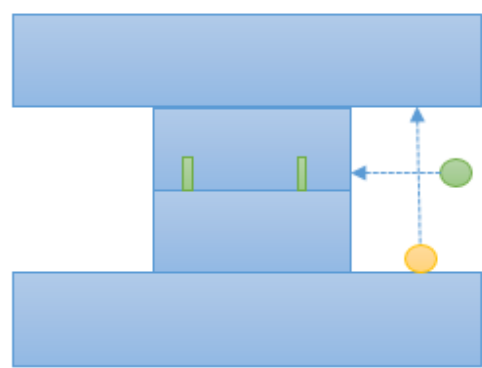

Figure 1. Procedure to Count Mold Shots with Photo Sensor and Distance Measurement Sensor

Figure 2 shows the algorithm of the developed module. In early stage, the module initializes, turning on three sensors. It turns on the Bluetooth connection to communicate with smart devices, enables the photo sensor to see whether the shaft is on the lower mold and initiates the distance measurement sensor to measure 
the distance between the upper mold and lower mold. The photo sensor detected the shaft, and upon detecting it, the distance measurement sensor measured the distance between the upper mold and lower mold. If the distance was greater than the pre-set maximum value, it signified that the upper mold did not move for a shot. On the other hand, a distance smaller than the pre-set minimum value meant that the upper mold moved for a shot. The module then added a shot and sent the number of shots to a tablet pc. The tablet pc in turn displayed the number to the worker, so that he can send it to the manager by transmitting it to the DB server through TCP/IP.

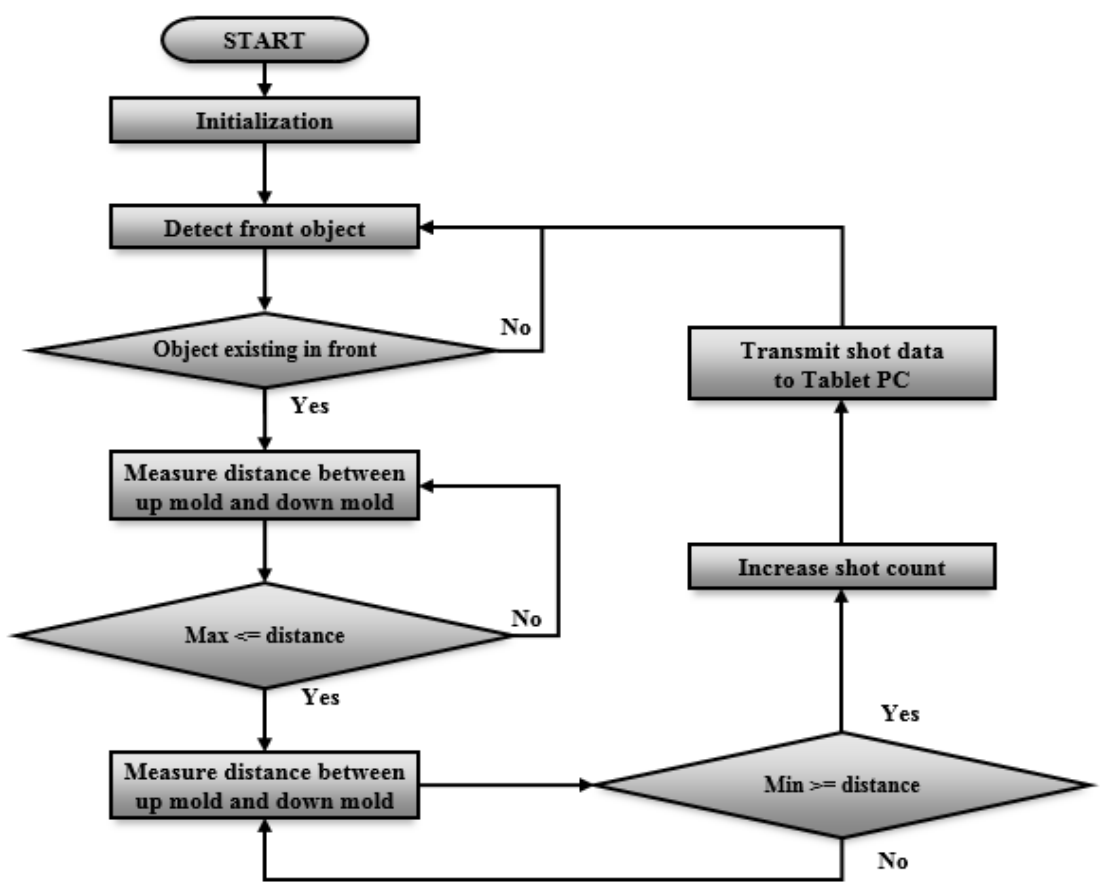

Figure 2. Algorithm of Life Cycle Management Module

\section{RESULTS AND ANALYSIS}

To analyze the performance of the new shot- management system, it was used in a local shaft-maker factory. The system comprised the developed module, photo sensor, distance measurement sensor and tablet pc. Using this system, it was tested whether the shots were accurately counted when the molding equipment injected the shaft.

Figure 3 displays the module and tablet PC installed to find out their applicability in an actual environment. Figure 3(a) shows the module developed by the study, mounted atmega128 MCU, Bluetooth-embedded module and LCD showing the shot count. Figure 3(b) shows the tablet pc installed in the molding equipment to present the actual shots, whose number was sent to the DB server through TCP/IP.

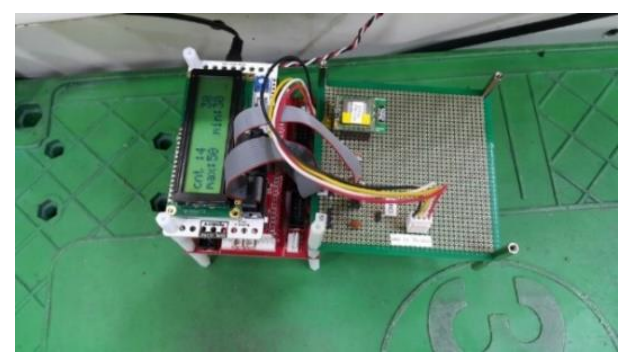

(a) Developed Module

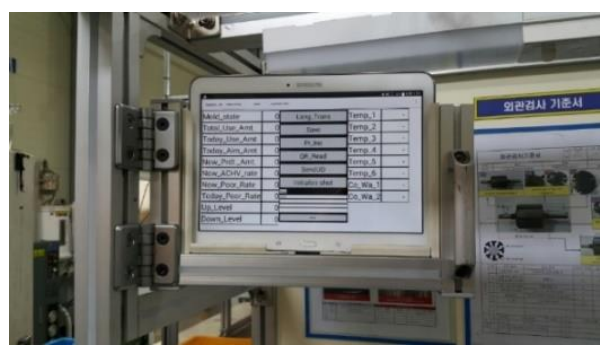

(b) Installed Teblet PC

Figure 3. Algorithm of Life Cycle Management Module 
Figure 4 compares the molding equipment before and after shots, where (a) is before shot and (b) is after shot. The photo sensor and the distance measurement sensor on the right side of the molding equipment informed whether there was a shaft and measured the distance between the upper mold and lower mold, respectively.

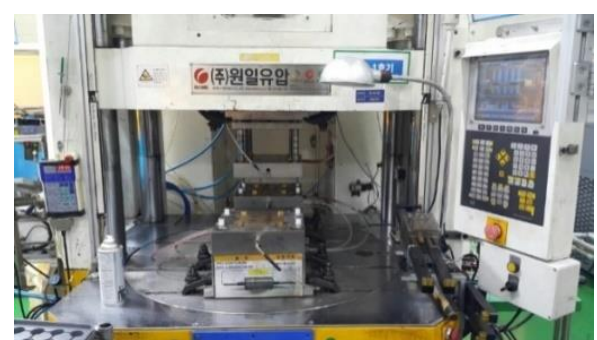

(a) Before Shot

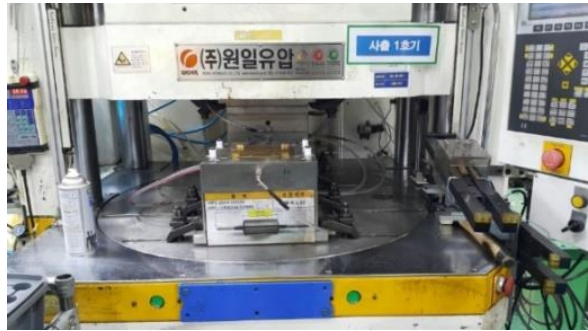

(b) After Shot

Figure 4. Photos of Molding Equipment before and after Shot

Figure 5 illustrates the module's shot result sent to the tablet pc via Bluetooth communication. The pc screen showed an increase in the number of shots, i.e. one shot in (a) and two shots in (b). The molding equipment produced four shafts per shot, meaning every extra shot led to four more productions. Besides, the maximum and minimum value can be transmitted through Bluetooth from the tablet pc to the module to designate the maximum and minimum values in Figure 2. The molding equipment produced injection with contact of upper mold and lower mold. Furthermore, the distance between the two molds varied depending up the type of equipment. The maximum and minimum values between the upper and the lower molds could be adjusted through the automatic mold shot measurement and management system. Therefore, our newly developed module can be applied to any molding equipment with various upper-lower gaps.

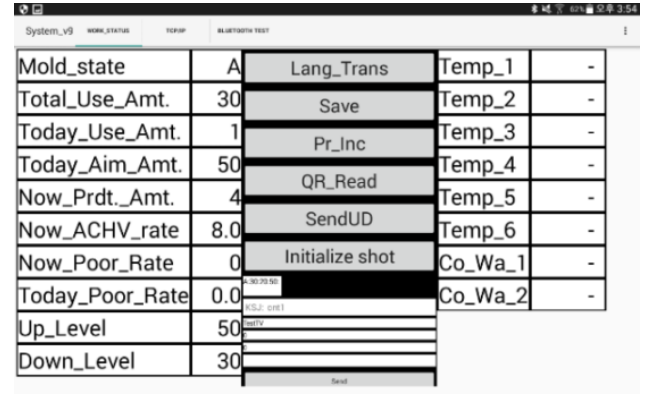

(a) Shot 1 Time

\begin{tabular}{|c|c|c|c|c|}
\hline 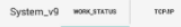 & mumom & & & \\
\hline Mold_state & A] & Lang_Trans & Temp_1 & - \\
\hline Total_Use_Amt. & 30 & Save & Temp_2 & - \\
\hline Today_Use_Amt. & 2 & Pr II C & Temp_3 & - \\
\hline Today_Aim_Amt. & 50 & - & Temp_4 & - \\
\hline Now_Prdt._Amt. & 8 & Qn_mead & Temp_5 & - \\
\hline Now_ACHV_rate & 16.0 & SendUD & Temp_6 & - \\
\hline Now_Poor_Rate & 0 & Initialize shot & Co_Wa_1 & - \\
\hline Today_Poor_Rate & 0.0 & & Co_Wa_2 & - \\
\hline Up_Level & 50 & & & \\
\hline Down_Level & 30 & & & \\
\hline
\end{tabular}

(b) Shot 2 Times

Figure 5. The Number of Shots Displayed on the Tablet pc Screen

\section{CONCLUSION}

Since increasing number of shots reduces the life cycle of mold equipment, shot management is necessary. To conclude, a module has been developed that can count the number of mold used. Such a system is extremely important for small and medium-sized enterprises (SMEs), and by applying it in the production line the SMEs will be able to manage the mold life cycle and improve product quality. The developed system uses a photo sensor, distance measurement sensor, Atmega128 MCU, tablet pc and Bluetooth communication module and includes the algorithm of life cycle management module to count shots. When the newly developed system was tested on a real molding equipment, results showed that the number of shots increased when the upper mold touched the lower mold. The maximum and minimum values between the upper and lower molds could be adjusted via the automatic mold shot measurement and management system. Therefore, the developed module can be applied to any molding equipment with various 
upper-lower gaps. In future studies, a system will be developed that will be able to send the number of shots from multiple injection equipment to the DB server using IoT.

\section{ACKNOWLEDGEMENTS}

The present Research has been conducted by the Graduate Research Project of KOREATECH in 2016.

\section{REFERENCES}

[1] P. Fallböhmer, et al., "Survey of The Die and Mold Manufacturing Industry - Practices in Germany, Japan, and the United States," Journal of Materials Processing Technology, vol. 59, pp. 158-168, 1996.

[2] K. Zhou, et al., "Industry 4.0: Towards Future Industrial Opportunities and Challenges," 2015 12th International Conference on Fuzzy Systems and Knowledge Discovery, pp. 2147-2152, 2015.

[3] S. Husain, et al., "Recent Trends in Standards Related to the Internet of Things and Machine-to-Machine Communications," Journal of Information and Communication Convergence Engineering, vol/issue: 12(4), pp. 228-236, 2014.

[4] K. C. Fan, "A Non-contact automatic measurement for free-form surface profiles," Computer Integrated Manufacturing Systems, vol/issue: 10(4), pp. 277-285, 1997.

[5] M. J. Tsai, et al., "Development of an Automatic Mold Polishing System," IEEE Transactions on Automation Science and Engineering, vol/issue: 2(4), pp. 393-397, 2005.

[6] J. Y. Lee and J. S. Oh, "A Tablet PC-based Monitoring System for Oceanic Applications," Journal of Information and Communication Convergence Engineering, vol/issue: 11(4), pp. 253-257, 2013.

[7] A. Thiriez and T. Gutowski, "An Environmental Analysis of Injection Molding," Proceedings of the 2006 IEEE International Symposium on Electronics and the Environment, pp. 195-200, 2006.

\section{BIOGRAPHIES OF AUTHORS}

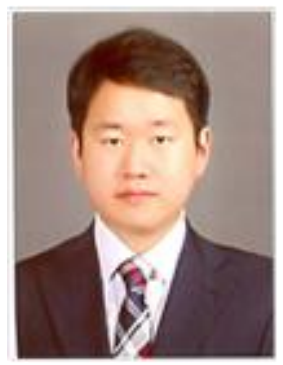

Hyun-Jun Shin. He received a B.S degree in the Department of electrical engineering from Namseoull university, Cheonan, Korea, in 2011, and an M.S. degree in Electrical, Electronics and Communication Engineering at the Korea University of Technology and Education, Cheonan, Korea, in 2011. He is currently pursuing a Ph.D. degree in Electrical, Electronics and Communication Engineering at the Korea University of Technology and Education, Cheonan, Korea. His research interests are in the industrial 4.0, smart factory, wireless sensor network, wireless localization, channel coding.

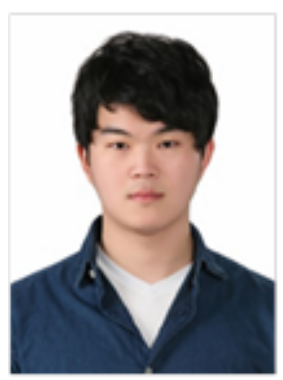

Sung-Jin Kim. He received a B.S degree in the Department of Information and Communication from Korea University of Technology and Education, Cheonan, Korea, in 2016. He is currently pursuing a M.S. degree in Electrical, Electronics and Communication Engineering at the Korea University of Technology and Education, Cheonan, Korea. His research interests are in the Bigdata.

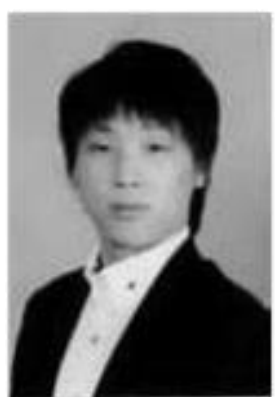

Min-Ho Jeon. He received the B.S degree in the Department of Game Digital Content from Far East University, Umsung, Korea, in 2009, and an M.S. degree in Electrical, Electronics and Communication Engineering at the Korea University of Technology and Education, Cheonan, Korea, in 2011. He is currently pursuing a Ph.D. degree in Electrical, Electronics and Communication Engineering at the Korea University of Technology and Education, Cheonan, Korea. His research interests are in the context-aware, wireless sensor network, wireless localization, channel coding and M2M network, Smart-Factory. 


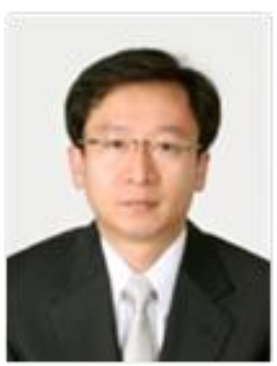

Chang-Heon Oh. He received the B. S. and M.S.E. degrees in telecommunication and information engineering from Korea Aerospace Univ. in 1988 and 1990, respectively. He received the Ph.D. degree in avionics engineering from Korea Aerospace Univ., in 1996. From Feb. 1990 to Aug. 1993, he was with Hanjin Electronics Co. From Oct. 1993 to Feb. 1999, he was with the CDMA R\&D center of Samsung Electronics Co. Since Mar. 1999, he has been with the School of Electrical, Electronics and Communication Engineering, Korea University of Technology and Education, where he is currently a professor. His research interests are in the areas of wireless communications, mobile communication, and wireless sensor networks with particular emphasis on wireless localization. 